\title{
Thermalization and ergodicity in one-dimensional many-body open quantum systems
}

\author{
Marko Žnidarič ${ }^{1}$ Tomaž Prosen, ${ }^{1}$ Giuliano Benenti, ${ }^{2,3}$ Giulio Casati, ${ }^{2,3,4}$ and Davide Rossini ${ }^{5}$ \\ ${ }^{1}$ Department of Physics, Faculty of Mathematics and Physics, University of Ljubljana, Ljubljana, Slovenia \\ ${ }^{2}$ CNISM, CNR-INFM, and Center for Nonlinear and Complex Systems, \\ Università degli Studi dell'Insubria, Via Valleggio 11, 22100 Como, Italy \\ ${ }^{3}$ Istituto Nazionale di Fisica Nucleare, Sezione di Milano, Via Celoria 16, 20133 Milano, Italy \\ ${ }^{4}$ Centre for Quantum Technologies, National University of Singapore, Singapore 117543 \\ ${ }^{5}$ International School for Advanced Studies (SISSA), Via Beirut 2-4, I-34014 Trieste, Italy
}

(Dated: November 8, 2018)

\begin{abstract}
Using an approach based on the time-dependent density-matrix renormalization group method, we study thermalization in spin chains locally coupled to an external bath. Our results provide evidence that quantum chaotic systems do thermalize, that is, they exhibit relaxation to an invariant ergodic state which, in the bulk, is well approximated by the grand canonical state. Moreover, the resulting ergodic state in the bulk does not depend on the details of the baths. On the other hand, for integrable systems we found that the invariant state in general depends on the bath and is different from the grand canonical state.
\end{abstract}

PACS numbers: 03.65.Yz, 75.10.Pq, 05.45.Mt

The emergence of canonical ensembles in quantum statistical mechanics from first principles is one of the key remaining old questions of theoretical physics. Even the definition of the temperature at the nano-scale poses a challenge [1]. Namely, the main question is how to "derive" the canonical distribution? It has been realized that the canonical distribution is in a way "typical": provided the overall system describing the environment plus a central system is in a generic pure state, the reduced state of the central system is with high probability canonical [2]. However, how precisely the canonical distribution arises from dynamical laws, without a priori statistical assumptions, is still unclear. Motivation in the study of this fundamental aspect of nonequilibrium physics also comes from some recent experiments with ultracold bosonic gases, where absence of thermalization in closed, integrable, strongly correlated quantum systems has been observed [3].

For closed many-body systems, integrability is believed to play a crucial role in the relaxation to the Steady State (SS): the nonequilibrium dynamics of a chaotic system is expected to thermalize at the level of individual eigenstates [4], as numerically observed in several physical models [5]. By contrast, for systems with non trivial integrals of motion, SSs usually carry memory of the initial conditions and are not canonical: maximizing the entropy while keeping the values of constants of motion fixed results in a generalized Gibbs ensemble [6]. Much less is known about the relaxation to the SS for open quantum systems [7]; this is what we are going to address in this paper. We provide numerical evidence that, analogously to closed systems, the occurrence of thermalization is strictly related to system's integrability, irrespective of the fine details of the baths. In particular we show that locally coupling a quantum chaotic many-body system to an environment is enough for a SS of the cen- tral system to be very close, in the bulk, to the canonical or grand canonical state (GCS). On the contrary, if the system is integrable, the constants of motion in general prevent thermalization and the form of the SS sensitively depends on the bath coupling operators. We show that the numerical description of an open quantum system in terms of a Lindblad equation with local coupling to the reservoirs is in some sense a computationally efficient, minimal model of thermalization. Such result paves the way for future simulations of quantum transport in large many-body quantum systems.

The time evolution for a generic state $\rho$ of an open quantum system can be described, under certain approximations, by a Lindblad master equation [8]:

$$
\frac{\mathrm{d}}{\mathrm{d} t} \rho=\frac{\mathrm{i}}{\hbar}[\rho, \mathcal{H}]+\hat{\mathcal{L}}_{\mathrm{B}} \rho
$$

where $\mathcal{H}$ is the Hamiltonian of the autonomous system, while the dissipation $\hat{\mathcal{L}}_{\mathrm{B}}=\gamma \sum_{k}\left(\left[L_{k} \rho, L_{k}^{\dagger}\right]+\left[L_{k}, \rho L_{k}^{\dagger}\right]\right)$ is parametrized by certain Lindblad operators $L_{k}$ (hereafter we set $\hbar=k_{B}=1$ and, unless noted otherwise, $\gamma=1)$. The derivation of Eq. (1) from first principles, i.e., from the Hamiltonian evolution of a system plus environment is rather tricky [8]; however it is the most general form of a completely positive, trace preserving, dynamical semi-group. Taking it for granted, we ask ourselves if, within this approximation, a finite many-body system can thermalize when coupled via some Lindblad operators $L_{k}$ acting only locally just on few degrees of freedom.

To elucidate the role covered by chaoticity in the thermalization process, we consider prototype onedimensional spin-1/2-chain models with nearest neighbor interactions: $\mathcal{H}=\sum_{l=0}^{n-2} h_{l, l+1}\left(h_{l, l+1}\right.$ denoting the local energy density, and $n$ being the chain length). As we shall see, the chosen models exhibit a crossover from integrable to chaotic regime when a suitable parameter in 
their Hamiltonians is varied. With the term "chaotic" we refer, as usual, to a system whose bulk energy spectrum of highly excited levels obeys a random matrix statistics [9]; in particular, the level spacing statistics (LSS) $p(s)$ is well approximated by the Wigner-Dyson distribution $p_{\mathrm{WD}}(s)$ [9], whereas in an integrable system LSS typically turns out to be Poissonian, $p_{\mathrm{P}}(s)$.

We assume local coupling to the reservoirs, i.e., the dissipator $\hat{\mathcal{L}}_{\mathrm{B}}$ acts only on the $m(\ll n)$ leftmost $(l)$ and rightmost $(r)$ spins: $\hat{\mathcal{L}}_{\mathrm{B}}=\hat{\mathcal{L}}_{\mathrm{B}}^{l} \otimes \hat{\mathbb{1}}_{\text {bulk }} \otimes \hat{\mathcal{L}}_{\mathrm{B}}^{r}$. We construct $\hat{\mathcal{L}}_{\mathrm{B}}$ by generalizing the method discussed in Ref. [10]. For this purpose, we first consider the GCS for the spin chain,

$$
\rho_{\mathcal{G}}(T, \mu)=Z^{-1} \exp \left[-\left(\mathcal{H}-\mu \Sigma^{z}\right) / T\right],
$$

where $\Sigma^{\mathrm{z}}=\sum_{l=0}^{n-1} \sigma_{l}^{\mathrm{z}}$ is the total magnetization $\left[\sigma_{j}^{\alpha}\right.$ ( $\alpha=\mathrm{x}, \mathrm{y}, \mathrm{z})$ being the Pauli operators for the $j$ th spin], $T$ the temperature, $\mu$ the "chemical potential", and $Z=\operatorname{tr}\left[\exp \left(-\left(\mathcal{H}-\mu \Sigma^{Z}\right) / T\right)\right]$ the partition function. Given a target temperature $T_{\text {targ }}$ and a chemical potential $\mu_{\mathrm{targ}}$, the reduced $m$-spin target density matrix $\rho_{\text {targ }}^{\lambda}, \lambda \in\{l, r\}$, is obtained after tracing $\rho_{\mathcal{G}}\left(T_{\text {targ }}, \mu_{\text {targ }}\right)$ over all but the $m$ leftmost/rightmost spins. We finally require that $\rho_{\text {targ }}^{\lambda}$ is the unique eigenvector of $\hat{\mathcal{L}}_{\mathrm{B}}^{\lambda}$ with eigenvalue 0 , while all other eigenvalues are equal to -1 . Such a choice produces, in absence of $\mathcal{H}$ and for a given spectral norm of $\hat{\mathcal{L}}_{\mathrm{B}}^{\lambda}$, the fastest convergence to $\rho_{\text {targ }}^{\lambda}$ [11]. In the presence of $\mathcal{H}$ we obtain, for up to $n \approx 100$ spins, the SS solution of Eq. (11) numerically by using a time-dependent Density Matrix Remormalization Group (tDMRG) method with a Matrix Product Operator (MPO) ansatz [12].

In the following we are interested in the asymptotic state reached, independently of initial conditions, after a long time, $\rho_{\mathrm{SS}} \equiv \lim _{t \rightarrow \infty} \rho(t)$. In all simulations we carefully checked that the simulation time was long enough to reach convergence, which is exponential. Since Lindblad operators act only locally and $\rho_{\mathcal{G}}(T, \mu)$ is invariant for the unitary part of Eq. (1), $\rho_{\mathrm{SS}}$ cannot be equal to the GCS, unless it is also an eigenstate of the dissipator $\hat{\mathcal{L}}_{\mathrm{B}}$. In other words, one can have $\rho_{\mathrm{SS}}=\rho_{\mathcal{G}}(T, \mu)$ only if $\rho_{\mathcal{G}}(T, \mu)=\rho_{\text {targ }}^{l} \otimes \rho_{\text {bulk }} \otimes \rho_{\text {targ }}^{r}$, i.e., if the GCS is separable with respect to the border $m$ spins which are used in the coupling. Nevertheless for chaotic systems, as we shall see, sufficiently far from the boundaries the state is arbitrarily close to $\rho_{\mathcal{G}}(T, \mu)$, regardless of the entanglement with the coupled parts.

Let us start our numerical investigations by considering a spin-1/2 Ising chain in a tilted magnetic field, described by the energy density

$$
h_{l, l+1}=J_{l} \sigma_{l}^{\mathrm{z}} \sigma_{l+1}^{\mathrm{z}}+\frac{b_{\mathrm{x}}}{2}\left(\sigma_{l}^{\mathrm{x}}+\sigma_{l+1}^{\mathrm{x}}\right)+\frac{b_{\mathrm{z}}}{2}\left(\sigma_{l}^{\mathrm{z}}+\sigma_{l+1}^{\mathrm{z}}\right) .
$$

Its only conserved quantity is the total energy, therefore the expected invariant state is the canonical one $\rho_{\mathcal{G}}(T, 0)$. To check thermalization, we solved the master equation

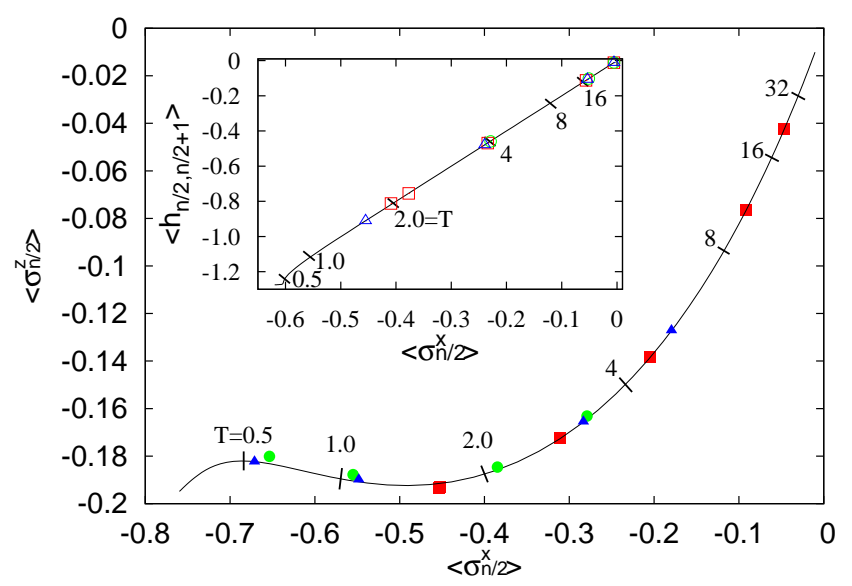

FIG. 1: (Color online). One-spin observables for the SS (symbols) agree with the theoretical canonical ones (full curve) to less than $0.5 \%$ for the chaotic Ising model (main plot). For the integrable model (inset) a comparable agreement is observed by looking at energy density (since $\left\langle\sigma_{n / 2}^{z}\right\rangle \equiv 0$ ) vs. $\left\langle\sigma_{n / 2}^{\mathrm{x}}\right\rangle$. Squares are for $n=16$ and uniform couplings; circles (triangles) for $n=16(n=40)$ and couplings $J_{l}$ switched on over a layer of thickness $\tau=4$, with $\gamma=0.2$. Marks on theoretical curves show the temperature.

for two different sets of parameters: (i) a transverse field $b_{\mathrm{x}}=1, b_{\mathrm{z}}=0$, for which the model is integrable and exhibits a Poissonian LSS; (ii) a tilted field $b_{\mathrm{x}}=1, b_{\mathrm{z}}=1$, for which it is chaotic with a Wigner-Dyson LSS 13] (if not specified, we take $J_{l}=1$ and couple two border spins, $m=2$ ). With the obtained $\rho_{\mathrm{SS}}$, we evaluated expectation values of several one- and two-spin observables in the bulk of the chain, and compared them to the theoretical ones as given by the canonical state $\rho_{\mathcal{G}}(T, 0)$.

In the main plot of Fig. 1 we show one-spin expectation values $\left\langle\sigma_{n / 2}^{\alpha}\right\rangle=\operatorname{tr}\left(\rho_{\mathrm{SS}} \sigma_{n / 2}^{\alpha}\right)$ for the chaotic case: all numerical points fall on the curve given by theoretical expectation values for a canonical state. The same happens in the integrable Ising model. Such irrelevance of integrability is a peculiarity of certain few-body observables, similarly to what observed in a different context of outof-equilibrium dynamics in closed systems 14. Quite remarkably, we could not reach temperatures in the bulk below $\approx 1.7$ (see squares in Fig. 11), even by using very small $T_{\text {targ }} \approx 0$. The reason resides in the already mentioned boundary effects due to entanglement between the boundary two spins and the bulk chain, which makes the cooling difficult. This must be contrasted with a zero attainable temperature in the case of separable states [15]. For entangled states though, our results show that to lower the minimal attainable temperature one has to reduce the effect of interaction at the boundaries which is responsible for entanglement. One way to do this is by switching on the interaction gently over a boundary layer of certain thickness $\tau, J_{l}=\sin \left(\frac{l}{\tau} \frac{\pi}{2}\right)\left(J_{n-2-l}=\sin \left(\frac{l}{\tau} \frac{\pi}{2}\right)\right)$, for $l=0, \ldots, \tau-1$, at the left (right) end and using a 


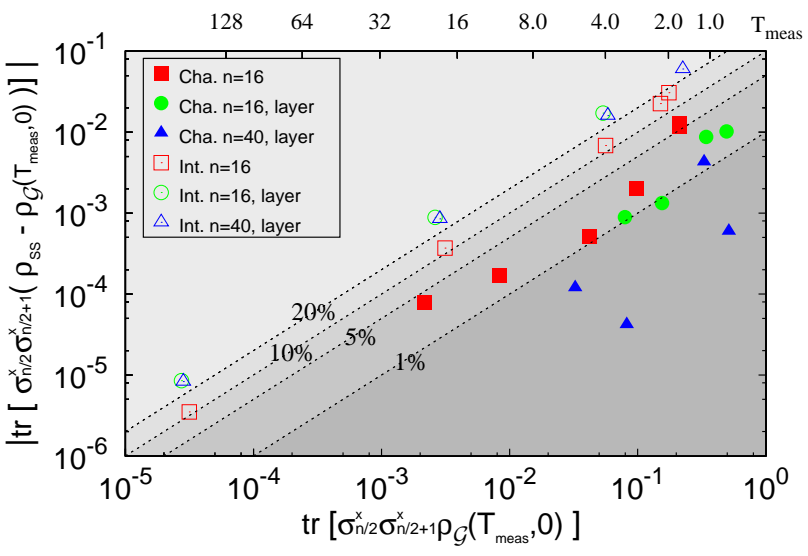

FIG. 2: (Color online). Absolute differences in the expectation value of a two-spin observable $\sigma_{n / 2}^{\mathrm{x}} \sigma_{n / 2+1}^{\mathrm{x}}$ between the SS and the theoretical canonical state, in the case of chaotic (full symbols) and integrable Ising model (empty symbols). Dashed lines denote constant relative error.

weaker coupling $\gamma$ (circles and triangles in Fig. 1).

To make comparison between $\rho_{\mathrm{SS}}$ and $\rho_{\mathcal{G}}(T, 0)$ quantitative, we determined the "measured" temperature $T_{\text {meas }}$ to which $\rho_{\mathrm{SS}}$ corresponds, which is in general different from $T_{\mathrm{targ}}$, due to boundary effects. Assuming that the $\mathrm{SS}$ is canonical in the bulk, one can extract $T_{\text {meas }}$ by comparing observables that uniquely set the temperature. For Ising model (3), the energy density is sufficient, therefore we used the condition $\operatorname{tr}\left[h_{n / 2-1, n / 2} \rho_{\mathrm{SS}}\right] \equiv$ $\operatorname{tr}\left[h_{n / 2-1, n / 2} \rho_{\mathcal{G}}\left(T_{\text {meas }}, 0\right)\right]$ to compute $T_{\text {meas }}$. We then calculated theoretical expectation values of other observables, through $\rho_{\mathcal{G}}\left(T_{\text {meas }}, 0\right)$; a comparison with the corresponding values for the reached SS may serve as an indicator of the quality of thermalization. In Fig. 2 we show differences between expectation values of $\sigma_{l}^{\mathrm{x}} \sigma_{l+1}^{\mathrm{x}}$, computed with $\rho_{\mathrm{SS}}$ and $\rho_{\mathcal{G}}\left(T_{\text {meas }}, 0\right)$, for both chaotic and integrable Ising chains. A marked distinction between the two cases appears. First, in the chaotic model errors are much smaller than in the integrable one; second, switching $J_{l}$ gradually, which should decrease errors due to smaller boundary effects, in the integrable case even worsens the situation. The integrable Ising model therefore does not relax to a canonical state in the bulk. Similar results are obtained for other few-spin observables, as well as for the lowest moments of the energy distribution: we evaluated $\left\langle\left[\left(\mathcal{H}_{6}-\left\langle\mathcal{H}_{6}\right\rangle\right) / 5\right]^{p}\right\rangle\left(p=2, \ldots, 5\right.$ and $\mathcal{H}_{6}$ is the Hamiltonian of the 6 central spins) on the states $\rho_{\mathrm{SS}}$ and $\rho_{\mathcal{G}}\left(T_{\text {meas }}, 0\right)$. In a chain of $n=40$ spins relative errors are never greater than $1 \%$ in the chaotic case, and are typically an order of magnitude larger in the integrable case.

To corroborate the importance of system's integrability on the convergence to invariant statistical ensembles, we consider another prototype model of interacting spins: the Heisenberg XXZ chain in a magnetic field, described
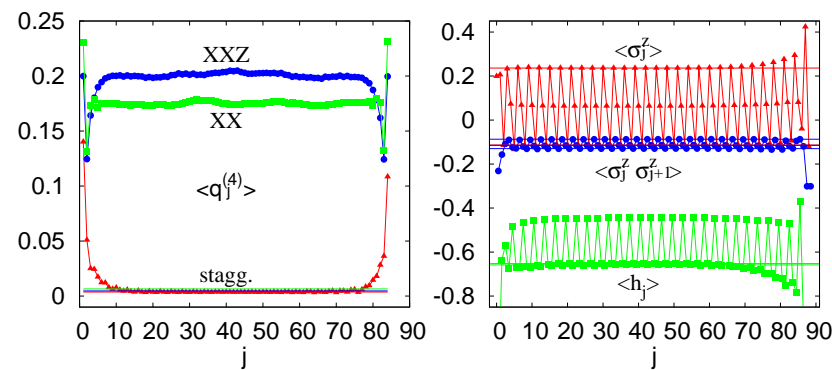

FIG. 3: (Color online). SS (symbols) and GCS (full lines) expectation values of $q_{j}^{(4)}$ (left panel), $\sigma_{j}^{\mathrm{z}}, \sigma_{j}^{\mathrm{z}} \sigma_{j+1}^{\mathrm{z}}$, and $h_{j}$ (right panel) for the Heisenberg model with $n=89$ spins, $T_{\text {targ }}=4, q_{\mathrm{targ}}=2, m=3, J_{l}=1$. In the left panel, "XX" and "XXZ" refer to two integrable cases without magnetic field (respectively at $\Delta=0,0.5$ ), "stagg." to the chaotic case with $\Delta=0.5$ and period-3 staggered field with $B=2$. The curves in the right panel are for the chaotic case only. The $\operatorname{GCS} \rho_{\mathcal{G}}\left(T_{\text {meas }}=5.851, \mu_{\text {meas }}=-0.534\right)$ for the chaotic case is obtained by matching $\left\langle h_{3 l+1,3 l+2}\right\rangle$ and $\left\langle\sigma_{3 l+1}^{\mathrm{z}}\right\rangle$ for which lines are not shown in the right panel.

by the energy density

$h_{l, l+1}=J_{l}\left(\sigma_{l}^{\mathrm{x}} \sigma_{l+1}^{\mathrm{x}}+\sigma_{l}^{\mathrm{y}} \sigma_{l+1}^{\mathrm{y}}+\Delta \sigma_{l}^{\mathrm{z}} \sigma_{l+1}^{\mathrm{z}}\right)+\frac{b_{l}}{2} \sigma_{l}^{\mathrm{z}}+\frac{b_{l+1}}{2} \sigma_{l+1}^{\mathrm{z}}$.

If the field is homogeneous the model is integrable and possesses, besides energy and magnetization, an infinite sequence of conserved quantities [16]. On the other hand, integrability can be broken, e.g., simply by means of a period-3 staggered magnetic field, $b_{3 k}=-B, b_{3 k+1}=$ $-B / 2, b_{3 k+2}=0$. In order to highlight the lack of thermalization in the integrable regime $B=0$, we target a non-Gibbsian state different from the GCS; namely, we use $\rho_{\text {non- } \mathcal{G}}(T, q) \sim \exp \left(-\mathcal{H} / T+q Q_{4}\right)$, with $Q_{4}=$ $-h_{0,1}-h_{n-2, n-1}+\sum_{l=0}^{n-4} q_{l}^{(4)}, q_{l}^{(4)}=\sigma_{l}^{\mathrm{x}} \sigma_{l+1}^{\mathrm{z}} \sigma_{l+2}^{\mathrm{z}} \sigma_{l+3}^{\mathrm{x}}+$ $\sigma_{l}^{\mathrm{y}} \sigma_{l+1}^{\mathrm{z}} \sigma_{l+2}^{\mathrm{z}} \sigma_{l+3}^{\mathrm{y}}$, being a conserved charge for an open chain with $\Delta=0$ and $b_{l}=0$ [16]. The idea is that, using a $q_{\mathrm{targ}} \neq 0$, in the integrable regime the SS exhibits strong deviations from the GCS, corresponding to $q=0$, while we expect chaotic dynamics to drive the bulk towards the GCS. Such expectation is confirmed by our numerical data. In Fig. 3 we show the spatial dependence of various observables for integrable, as well as for chaotic cases. In the integrable cases deviations from the GCS expectations are large, while they become very small for a chaotic system. Analogously to the Ising model, we checked this statement also for other few-spin observables (we found that, in presence of chaos, the largest discrepancy among all the one- and two-spin observables amounts to $2 \times 10^{-4}$ ); layered interactions in the integrable model do not help in thermalizing the system.

A further confirmation of the role of integrability comes from a direct analysis of the quality of thermalization after gradually switching on the perturbation that 


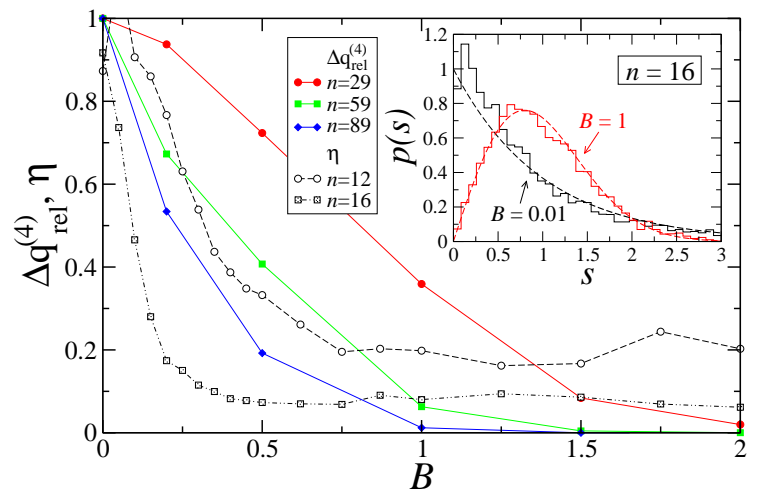

FIG. 4: (Color online). Relative differences $\Delta q_{\mathrm{rel}}^{(4)}=$ $\Delta q^{(4)}(B) / \Delta q^{(4)}(0)$ in $q^{(4)}$ expectation values on the SS and the GCS evaluated in the bulk of the Heisenberg model with $\Delta=0.5$, as the staggering strength $B$ is varied (full curves). Also shown is dependence of the $\eta$ function (dashed curves), characterizing the integrable-chaotic crossover. For both quantities the crossover takes place at smaller $B$ with increasing $n$. Inset: two examples of LSS in the integrable $(B=0.01)$ and chaotic $(B=1)$ regimes; dashed lines denote Poissonian and Wigner-Dyson statistics [9], respectively.

drives the crossover from integrability to chaos: the longitudinal field $b_{z}$ in Eq. (3) or the staggering intensity $B$ in Eq. (4). As shown in Fig. 4 for the Heisenberg model, such crossover is conveniently detected by the parameter $\eta \equiv \int\left|p(s)-p_{\mathrm{WD}}(s)\right| \mathrm{d} s / \int\left|p_{\mathrm{P}}(s)-p_{\mathrm{WD}}(s)\right| \mathrm{d} s ; \eta=1$ and $\eta=0$ correspond to Poissonian and Wigner-Dyson distributions, respectively. In the same figure we also plot deviations in $\left\langle q^{(4)}\right\rangle$ evaluated on the SS and on the corresponding GCS: $\Delta q^{(4)}=\operatorname{tr}\left[q_{l}^{(4)}\left(\rho_{\mathrm{SS}}-\rho_{\mathcal{G}}\left(T_{\text {meas }}, \mu_{\text {meas }}\right)\right)\right]$ as the strength $B$ of the staggered magnetic field is increased. The progressive onset of chaos gradually improves the quality of thermalization, being $\Delta q^{(4)}$ a monotonic decreasing function of $B$. Moreover, the strength of the staggered field required to converge to the GC expectation value drops with the system size.

In conclusion, we have shown that, within the Lindblad equation formalism, coupling a one-dimensional quantum chaotic system locally to a bath results in a SS being equal to the invariant (grand)canonical state, far away from the coupled sites. In contrast, integrable systems do not thermalize and their SSs exhibit strong deviations from the (grand)canonical state, depending on the details of the coupling. The fact that for chaotic systems the SS does not depend on the details of the coupling, shows that very likely the same result would be obtained even for a harder-to-treat Hamiltonian evolution of a system plus environment or for higher dimensional systems. Our method should be applicable also to non-equilibrium situations. Indeed, by locally coupling a system to two or several baths at different values of temperature and chemical potentials, one should be able to efficiently con- trol local thermalization. Thus, our results might open significant new perspectives in the simulation of quantum transport in many-body quantum systems in contact with thermal and chemical baths.

We thank V. Giovannetti for useful discussions. MŽ and TP are supported by the Program P1-0044, and the Grant J1-2208, of the Slovenian Research Agency.

[1] M. Hartmann et al., Phys. Rev. Lett 93, 080402 (2004).

[2] H. Tasaki, Phys. Rev. Lett. 80, 1373 (1998); J. Gemmer and G. Mahler, Eur. Phys. J. B 31, 249 (2003); S. Goldstein et al., Phys. Rev. Lett. 96, 050403 (2006); S. Popescu et al., Nat. Phys. 2, 754 (2006); P. Reimann, Phys. Rev. Lett. 99, 160404 (2007). N. Linden et al., Phys. Rev. E 79, 061103 (2009).

[3] T. Kinoshita et al., Nature 440, 900 (2006); S. Hofferberth et al., Nature 449, 324 (2007).

[4] J. M. Deutsch, Phys. Rev. A 43, 2046 (1991); M. Srednicki, Phys. Rev. E 50, 888 (1994); V. V. Flambaum and F. M. Izrailev, Phys. Rev. E 56, 5144 (1997).

[5] S. Åberg, Phys. Rev. Lett. 64, 3119 (1990); Ph. Jacquod and D. L. Shepelyansky, Phys. Rev. Lett. 79, 1837 (1997); G. Benenti et al., Eur. Phys. J D 17, 265 (2001); C. Kollath et al., Phys. Rev. Lett. 98, 180601 (2007); S. R. Manmana et al., Phys. Rev. Lett. 98, 210405 (2007); M. Rigol et al., Nature 452, 854 (2008); M. Rigol, Phys. Rev. Lett. 103, 100403 (2009); S. Yuan et al., J. Phys. Soc. Jpn. 78, 094003 (2009).

[6] M. Rigol et al., Phys. Rev. Lett. 98, 050405 (2007); M. A. Cazalilla, Phys. Rev. Lett. 97, 156403 (2006); D. C. Brody et al., J. Phys. A. 40, F503 (2007); T. Barthel and U. Schollwöck, Phys. Rev. Lett 100, 100601 (2008); M. Eckstein and M. Kollar, Phys. Rev. Lett. 100, 120404 (2008); D. M. Gangardt and M. Pustilnik, Phys. Rev. A 77, 041604(R) (2008); M. Kollar and M. Eckstein, Phys. Rev. A 78, 013626 (2008).

[7] M. J. Henrich et al., Phys. Rev. E 72, 026104 (2005); D. Patanè et al., Phys. Rev. Lett. 102, 245701 (2009).

[8] H.-P. Breuer and F. Petruccione, The Theory of Open Quantum Systems (Oxford University Press, Oxford, 2002); R. Alicki and K. Lendi, Quantum Dynamical Semigroups and Applications (Springer, Berlin, 2007).

[9] F. Haake, Quantum Signatures of Chaos (Springer, Berlin, 2001).

[10] T. Prosen and M. Žnidarič, J. Stat. Mech. P02035 (2009).

[11] To construct such $\hat{\mathcal{L}}_{\mathrm{B}}^{l}$ (and similarly for $\hat{\mathcal{L}}_{\mathrm{B}}^{r}$ ), we first diagonalize $\rho_{\text {targ }}^{l}$, thus getting eigenvalues $d_{i}$ and eigenvectors, $\rho_{\text {targ }}^{l}=V^{\dagger} d V$. Then we obtain $\hat{\mathcal{L}}_{\mathrm{B}}^{l}$ after rotating the "diagonal" Lindblad superoperator $\hat{\mathcal{L}}_{\mathrm{B}}^{\text {diag }}$, $\hat{\mathcal{L}}_{\mathrm{B}}^{l}=R \hat{\mathcal{L}}_{\mathrm{B}}^{\text {diag }} R^{\mathrm{T}}$, with the orthogonal matrix $R_{\underline{\alpha}, \underline{\beta}}=$ $\operatorname{tr}\left(V^{\dagger} \sigma^{\underline{\alpha}} V \sigma^{\underline{\beta}}\right) / 4\left(\sigma^{\underline{\alpha}}=\sigma^{\alpha_{1}} \otimes \cdots \otimes \sigma^{\alpha_{m}}\right)$. A set of $4^{m}$ Lindblad operators leading to the diagonal superoperator $\hat{\mathcal{L}}_{\mathrm{B}}^{\text {diag }}$ is given by $L_{i_{1} i_{2} \ldots i_{m}}=\sqrt{\frac{d_{k}}{2 \cdot 4^{m}}} r^{i_{1}} \otimes r^{i_{2}} \otimes \cdots \otimes r^{i_{m}}$, with $i_{j} \in\{0,1,2,3\}, k=\sum_{j=1}^{m}\left(i_{j} \bmod 2\right) \cdot 2^{j-1}$, and $r^{0}=\sigma^{\mathrm{x}}+\mathrm{i} \sigma^{\mathrm{y}}, r^{1}=\sigma^{\mathrm{x}}-\mathrm{i} \sigma^{\mathrm{y}}, r^{2}=\mathbb{1}+\sigma^{\mathrm{z}}, r^{3}=\mathbb{1}-\sigma^{\mathrm{z}}$.

[12] See, e.g., A. J. Daley et al., J. Stat. Mech. P04005 (2004); F. Verstraete et al., Adv. Phys. 57, 143 (2008). We im- 
plemented the propagator corresponding to Eq. (1) in small time steps of length $d t=0.05$; for each of them we used a 4th-order Trotter expansion.

[13] T. Prosen and M. Žnidarič, Phys. Rev. E 75, 015202 (2007).

[14] D. Rossini et al., Phys. Rev. Lett. 102, 127204 (2009).

[15] D. Burgarth and V. Giovannetti, Phys. Rev. Lett. 99,
100501 (2007). We checked that, for separable ground states (e.g., in the ferromagnetic Heisenberg chain), boundary effects are absent and one can cool to $T=0$.

[16] M. P. Grabowski and P. Mathieu, J. Phys. A 29, 7635 (1996). 